\title{
Anti-Unruh effect in the thermal background
}

\author{
Yongjie Pan® and Baocheng Zhang®* \\ School of Mathematics and Physics, China University of Geosciences, Wuhan 430074, China
}

(Received 1 October 2021; accepted 29 November 2021; published 16 December 2021)

\begin{abstract}
We study the influence of the thermal background on the existence of the anti-Unruh effect. For the massless scalar field, we present that the anti-Unruh effect can appear when the detector is accelerated in the thermal field, which is shown to be absent when accelerating in the Minkowski vacuum. For the massive scalar field, it is found that the anti-Unruh effect which exists in the case for accelerated detectors in the vacuum can disappear when the background temperature increases. We use the many-body entangled quantum state to study the situation of the massive scalar field. When the many-body state is accelerated, its entanglement is decreasing with the increase of the background temperature and the phenomenon of the sudden death for entanglement occurs. This provides a valuable indication that the anti-Unruh effect can exist below some specific background temperature, dependent on the actual experimental environment.
\end{abstract}

DOI: 10.1103/PhysRevD.104.125014

\section{INTRODUCTION}

The Unruh effect [1] was discovered in 1976, which states that an observer with uniform acceleration would feel a thermal bath of particles in the Minkowski vacuum of a free quantum field. Since then, the effect had been digested and extended to many different situations (see the review [2] and references therein). Although many proposals based mainly on the Unruh-DeWitt (UDW) detector [3] had been put forward [4-16] in past years, the observation of Unruh effect has not been realized up to now, because of the pretty low Unruh temperature which requires the acceleration to be about $10^{20} \mathrm{~m} / \mathrm{s}^{2}$ in order to realize a photon bath at $1 \mathrm{~K}$. As is well known, an elemental obstacle to test the Unruh effect experimentally is the thermal noise from the environment. The recent found anti-Unruh effect [17] provides a helpful way to test the effect experimentally, since it usually leads to the different behaviors from that by the thermal noise. This phenomena of anti-Unruh effect follows from the observation [17] that an increase in detector acceleration will correspond to a decrease in the temperature of the detected radiation, in direct contrast to both the Unruh effect and one's expectations. In particular, the antiUnruh effect has been shown to represent a general stationary mechanism that can exist under a stationary state satisfying Kubo-Martin-Schwinger (KMS) condition

\footnotetext{
*zhangbc.zhang@yahoo.com
}

Published by the American Physical Society under the terms of the Creative Commons Attribution 4.0 International license. Further distribution of this work must maintain attribution to the author(s) and the published article's title, journal citation, and DOI. Funded by SCOAP.
[18-20] and is independent on any kind of boundary conditions [21]. Thus, like the Unruh effect, the antiUnruh effect constitutes another new phenomenon for the accelerated observers.

Since the experiments are always made in the range of finite length and time, it must distinguish the two situations of Unruh and anti-Unruh effects carefully. An interesting way for this is to see the change of quantum entanglement by acceleration. According to the previous results [22-30], the quantum entanglement would be degraded by the Unruh effect, which is similar to the results caused by the thermal background. However, a recent calculation showed that the anti-Unruh effect can lead to the increase for the quantum entanglement for the bipartite [31] and many-body quantum states [32].

The previous studies was mostly made for the change of quantum states and entanglement when the physical systems were accelerated in the Minkowski vacuum. As the thermal noise cannot be eliminated completely, it is significant to investigate the influence of acceleration on the quantum states or entanglement in the thermal background [33-37]. Since it is difficult to distinguish the physical results by the Unruh effect from that by the thermal background [38-40], we will investigate the influence of anti-Unruh effect on many-body entanglement and study whether it could be differentiated from thermal background in this paper. We will consider the spin squeezed states $[41,42]$ as done before in Ref. [32] but accelerated in the thermal background instead of the vacuum background. This is necessary because for accelerated observers in thermal field the Green functions which are essential in calculating the transition probability are not time translational invariant and the accelerated observers see the thermal background not in thermal 
equilibrium $[39,40]$. Thus, whether the earlier investigation of the anti-Unruh effect for accelerated many-body quantum systems in vacuum is still hold in the thermal background is unclear both in mathematical and physical perspective. This constitutes the main motivation for the present work. In this paper, we not only investigate the influence of background thermal field on the existence of anti-Unruh effect, but also we will study the influence on the accelerated many-body quantum systems. We will focus on twin-Fock (TF) states [43] which can be seen as a kind of limit for spin squeezed states and had been realized in a recent experiment with more than $10^{4}$ atoms [44].

This paper is organized as follows. In the second section we review the two-level Unruh-DeWitt (UDW) detector model, and investigate whether the anti-Unruh effect appears for the environment of the vacuum or the thermal field with the condition of the massless scalar field. Then, the model for the atom accelerated in the thermal field is given in the third section. This is followed in the fourth section by the discussions on the influence of acceleration on entanglement for TF states in the thermal background, where the spin squeezing parameter is used to measure the change of entanglement. Then, the phase sensitivity is analyzed for the accelerated TF states in the thermal background with the actual experimental conditions in Sec. V. Finally, we give a conclusion in Sec. VI. In this paper, we use units with $c=\hbar=k_{B}=1$, except the part of analyzing the phase sensitivity in Sec. V.

\section{THERMAL FIELD}

In this section we will investigate the influence of thermal field on existence of anti-Unruh effect qualitatively. Start from the model of the UDW detector. It is usually considered as a pointlike two-level quantum system or atom (as required in this paper) and consists of two quantum states, i.e., the ground $|g\rangle$ and excited $|e\rangle$ states, which are separated by an energy gap $\Omega$ while experiencing accelerated motion in a vacuum field. But for the accelerated atom, the Unruh effect will influence the state of the atom. This could be described according to the following interaction Hamiltonian in a $(1+1)$-dimension model, $H_{I}=\lambda \chi(\tau / \sigma) \mu(\tau) \phi(x(\tau))$, where $\phi$ is a minimally coupled scalar field related to the thermal background in Minkowski spacetime and interacts with the accelerated atom, $\lambda$ is the coupling strength, $\tau$ is the atom's proper time along its trajectory $x(\tau), \mu(\tau)$ is the atom's monopole momentum, and $\chi(\tau / \sigma)$ is a switching function that is used to control the interaction timescale $\sigma$. Thus, the total excitation probability for an accelerated detector initially in the ground state and evolving in the thermal background characterized by a temperature $\beta^{-1}=T$ can be expressed as $[33,36]$

$P_{+}=C \int d \tau \int d \tau^{\prime} \chi\left(\frac{\tau}{\sigma}\right) \chi\left(\frac{\tau^{\prime}}{\sigma}\right) e^{-i \Omega\left(\tau-\tau^{\prime}\right)} G_{\beta}^{+}\left(\tau, \tau^{\prime}\right)$

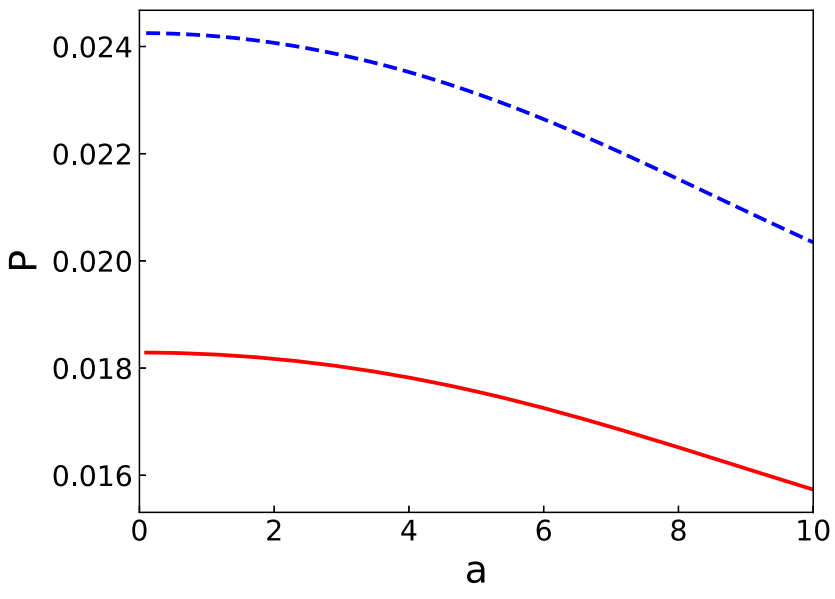

FIG. 1. The transition probability as a function of the acceleration $a$. The model parameters employed are $\lambda=1$, $\sigma=0.2, \Omega=0.3$. The solid red line denotes the case in which the switching function is Gaussian, and the dashed blue line denotes the case in which the switching function is rectangular due to the finite interaction time.

which is calculated only at the leading order with $C=$ $\lambda^{2}|\langle e|\mu(0)| g\rangle|^{2} . \quad G_{\beta}^{+}\left(\tau, \tau^{\prime}\right) \equiv G_{\beta}^{+}\left(x(\tau), x\left(\tau^{\prime}\right)\right)=\langle\phi(x(\tau)) \times$ $\left.\phi\left(x\left(\tau^{\prime}\right)\right)\right\rangle_{\beta}$ is the thermal Green function by taking the Gibbs ensemble average. When $T=0, G_{\beta}^{+}\left(\tau, \tau^{\prime}\right)=$ $G_{0}^{+}\left(\tau, \tau^{\prime}\right)$ which is the vacuum Green function for accelerating atom in the vacuum.

At first, we make a little analysis about the influence of the time transitional invariance on the existence of antiUnruh effect. As known in Ref. [21,39] that $G_{0}^{+}\left(\tau, \tau^{\prime}\right)=$ $G_{0}^{+}\left(\tau-\tau^{\prime}\right)$ which indicates that the vacuum Green function is time transitional invariant, but the entire integrand $F_{0}\left(\tau, \tau^{\prime}\right)=\chi\left(\frac{\tau}{\sigma}\right) \chi\left(\frac{\tau^{\prime}}{\sigma}\right) e^{-i \Omega\left(\tau-\tau^{\prime}\right)} G_{0}^{+}\left(x(\tau), x\left(\tau^{\prime}\right)\right)$ is not invariant because the switching function takes the Gauss form, $\chi\left(\frac{\tau}{\sigma}\right)=\exp \left[-\frac{\tau^{2}}{2 \sigma^{2}}\right]$. We can choose the proper switching function to preserve the time transitional invariance for the integrand $F_{0}\left(\tau, \tau^{\prime}\right)$, i.e., $\chi\left(\frac{\tau}{\sigma}\right)=1$. From the Fig. 1 , it is seen that the anti-Unruh effect exists for the two situations, which shows at least that the time transitional invariance of the vacuum Green function is not conclusive element for the existence of the anti-Unruh effect. Furthermore, it might imply that the anti-Unruh effect could exist in the thermal background even though the thermal Green function is not time transitional invariance. In this paper, we will show this point by two different aspects. The one is to present that the anti-Unruh effect could appear in thermal background but it does not exist in the vacuum for the massless scalar field. Another one is to show that the anti-Unruh effect exists in the vacuum for the massive scalar field but whether it still exists in thermal background depends on the temperature of the background field. The latter one will be studied in the next section with the many-body quantum system, and the former one will be analyzed here qualitatively. 
When the accelerated atom moves along the trajectory,

$$
\begin{aligned}
& t(\tau)=a^{-1} \sinh (a \tau), \\
& x(\tau)=a^{-1} \cosh (a \tau),
\end{aligned}
$$

with the proper acceleration $a$, ones can calculate the Green functions as $[33,40]$

$$
G_{0}^{+}(\tau, 0)=-\frac{a^{2}}{16 \pi^{2}} \frac{1}{\sinh ^{2}(a \tau / 2)},
$$

for the vacuum, and

$$
\begin{aligned}
G_{\beta}^{+}(\tau, 0)= & \frac{a}{16 \pi \beta \sinh ^{2}(a \tau / 2)}\left[\operatorname{coth}\left(\frac{\pi}{a \beta}\left(e^{a \tau}-1\right)\right)\right. \\
& \left.-\operatorname{coth}\left(\frac{\pi}{a \beta}\left(1-e^{-a \tau}\right)\right)\right],
\end{aligned}
$$

for the thermal field. Here we take $\tau^{\prime}=0$ for simplicity. Note that the breakdown of the time transitional invariance in Eq. (4) cannot be seen clearly. It does not matter for the analysis of the anti-Unruh effect, as pointed out above. Moreover, the thermal Green function (4) is calculated using massless scalar field, and will reduce to the vacuum result (3) as the background temperature approaches to zero or $\beta \rightarrow \infty$.

According to Ref. [21], the anti-Unruh effect exists under the weak condition, $\frac{\partial P_{+}}{\partial a}<0$, base on such concept for antiUnruh effect: the transition probability of an accelerated detector can actually decrease with acceleration. As analyzed above, the switching function is not crucial for the existence of the anti-Unruh effect. Thus, we ignore the switching function here for the further analysis. This means that $P_{+} \sim C \int d \tau e^{-i \Omega \tau} G^{+}(\tau, 0) \sim C \tilde{G}(\Omega)$ where $\tilde{G}(\Omega)$ is the Fourier transform of $G^{+}(\tau, 0)$. Therefore, the weak condition for the anti-Unruh effect becomes $\frac{\partial \tilde{G}(\Omega)}{\partial a}<0$.

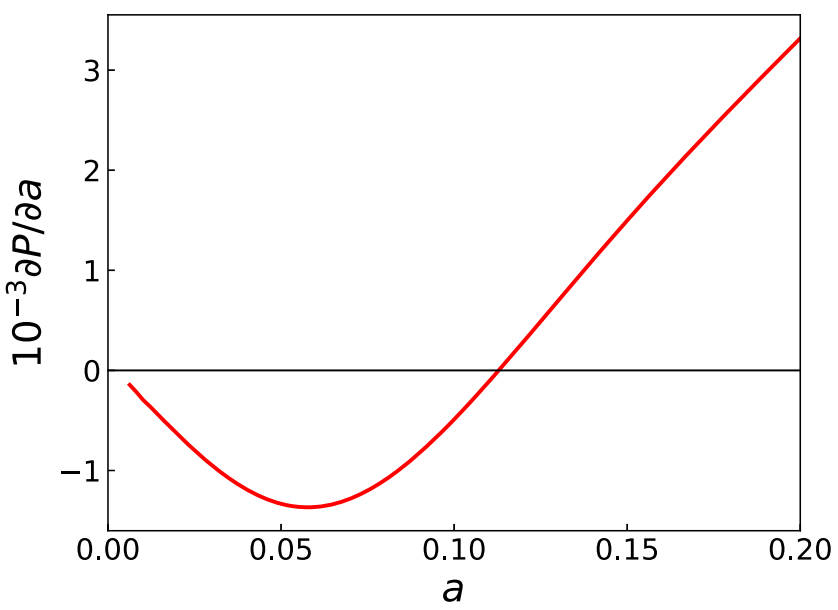

FIG. 2. The derivative of the transition probability with respect to the acceleration as a function of the acceleration $a$. The inverse of the background temperature is taken as $\beta=30$, and the other parameters are the same as in Fig. 1.
For the acceleration in the vacuum, we have

$$
\tilde{G}_{0}(\Omega)=\frac{a+\pi \Omega \operatorname{coth}(\pi \Omega / a)}{2 \pi^{5 / 2}} .
$$

It is not hard to show that $\frac{\partial \tilde{G}_{0}(\Omega)}{\partial a}>0$ for any $a$ or $\Omega$. This is just the result in Ref. [21] that the anti-Unruh effect is absent for accelerated detectors in the Minkowski vacuum of a massless scalar field.

For the acceleration in the thermal field, we can calculate the transition probability as in the case for the acceleration in the vacuum. However, there is no analytical expression for the Fourier transform of $G_{\beta}^{+}(\tau, 0)$ in Eq. (4). Therefore, the numerical result is given in Fig. 2, in which it is seen that anti-Unruh effect appears for the small acceleration. This shows that the introduction of thermal field would change the influence of acceleration on the quantum transition process. In the next section, we will discuss that the influence of thermal field on the change of quantum states or entanglement led by acceleration with the manybody physical systems.

\section{ACCELERATION IN THERMAL FIELD}

We still consider the UDW model for the single atom and the interaction unitary operation $U=I-i \int d \tau H_{I}(\tau)+$ $O\left(\lambda^{2}\right)$ is expanded to the first order. As the atoms are accelerated in thermal background, the evolution of the accelerated atom can be written with the density operators as

$$
\rho_{f}=U^{\dagger} \rho_{i} U
$$

where the initial density operator consists of the product form of the density operator for the atom and the density operator for the thermal field, $\rho_{t h}=\sum_{n} p_{n}|n\rangle\langle n|$ with $p_{n}=$ $\left(1-e^{-\beta \omega}\right) e^{-n \beta \omega}$. Thus, within the first-order approximation and in the interaction picture, the evolution of the atom could be described by

$$
\begin{aligned}
\operatorname{Tr}_{t h}\left[U^{\dagger}\left(\rho_{a 1} \otimes \rho_{t h}\right) U\right] & =\sum_{n} p_{n}\left(|g\rangle\left\langle\left. g|+n| u_{+}\right|^{2} \mid e\right\rangle\langle e|\right), \\
\operatorname{Tr}_{t h}\left[U^{\dagger}\left(\rho_{a 2} \otimes \rho_{t h}\right) U\right] & =\sum_{n} p_{n}|g\rangle\langle e|, \\
\operatorname{Tr}_{t h}\left[U^{\dagger}\left(\rho_{a 3} \otimes \rho_{t h}\right) U\right] & =\sum_{n} p_{n}|e\rangle\langle g|, \\
\operatorname{Tr}_{t h}\left[U^{\dagger}\left(\rho_{a 4} \otimes \rho_{t h}\right) U\right] & =\sum_{n} p_{n}\left((n+1)\left|u_{-}\right|^{2}|g\rangle\langle g|+| e\rangle\langle e|\right),
\end{aligned}
$$

where $\lambda=1$ is taken, $\operatorname{Tr}_{t h}$ represents the calculation of tracing out the field degrees of freedom, and the normalization factors are not written but are considered in the following calculations for the plots. The initial density operators for the atom are taken as $\rho_{a 1}=|g\rangle\langle g|, \rho_{a 2}=$ $|g\rangle\left\langle e\left|, \rho_{a 3}=\right| e\right\rangle\left\langle g\left|, \rho_{a 4}=\right| e\right\rangle\langle e|$. These expressions in Eq. (7) provide the elementary forms of the evolution for 
the general state of the atoms. Since an atom is only considered to have two levels of energy, the change of the field should be limited within these basis of $\{|n-1\rangle$, $|n\rangle,|n+1\rangle\}$, dependent on the absorption or emission of the photon from the field by the atom. $u_{ \pm}=$ $\sum_{k} \int_{-\infty}^{\infty} \chi(\tau / \sigma) \exp \left[ \pm i \Omega \tau+i \omega_{k} t(\tau)-i k x(\tau)\right] d \tau$ are related to the motion of the atom. With the trajectory of the atom in Eq. (2), the transition probability can be calculated according to

$$
\begin{aligned}
P_{+} & =\left\langle n-1\left|\left\langle e\left|U^{\dagger}\left(\rho_{a 1} \otimes \rho_{t h}\right) U\right| e\right\rangle\right| n-1\right\rangle \\
& =\sum_{n} n p_{n}\left|u_{+}\right|^{2} .
\end{aligned}
$$

Comparing it with Eq. (1), it is not difficult to confirm their consistency by taking the field $\phi(x(\tau))=$ $\sum_{k} \frac{e^{-i k x(\tau)}}{\sqrt{2 \omega_{k}}}\left(a_{k} e^{-i \omega_{k} t(\tau)}+a_{k}^{\dagger} e^{i \omega_{k} t(\tau)}\right)$ where $k$ denotes the mode of the $(1+1)$-dimension scalar field with (bosonic) annihilation (creation) operator $a_{k}\left(a_{k}^{\dagger}\right), a_{k}|n\rangle=\sqrt{n}|n-1\rangle$ and $a_{k}^{\dagger}|n\rangle=\sqrt{n+1}|n+1\rangle$. Note that $P_{+}$represents the transition probability from the ground state to the excitation state. Similarly, the transition probability $P_{-}$from the excitation state to the ground state can be calculated as $P_{-}=\langle n+$ $\left.1\left|\left\langle g\left|U^{\dagger}\left(\rho_{a 4} \otimes \rho_{t h}\right) U\right| g\right\rangle\right| n+1\right\rangle=\sum_{n}(n+1) p_{n}\left|u_{-}\right|^{2}$.

It was pointed out that the change of the quantum state, i.e., the transition probability, is dependent on the concrete parameters like the interaction time scale $\sigma$ and the energy gap $\Omega$ [17]. The probability would decrease as the acceleration or the Unruh temperature increases under some

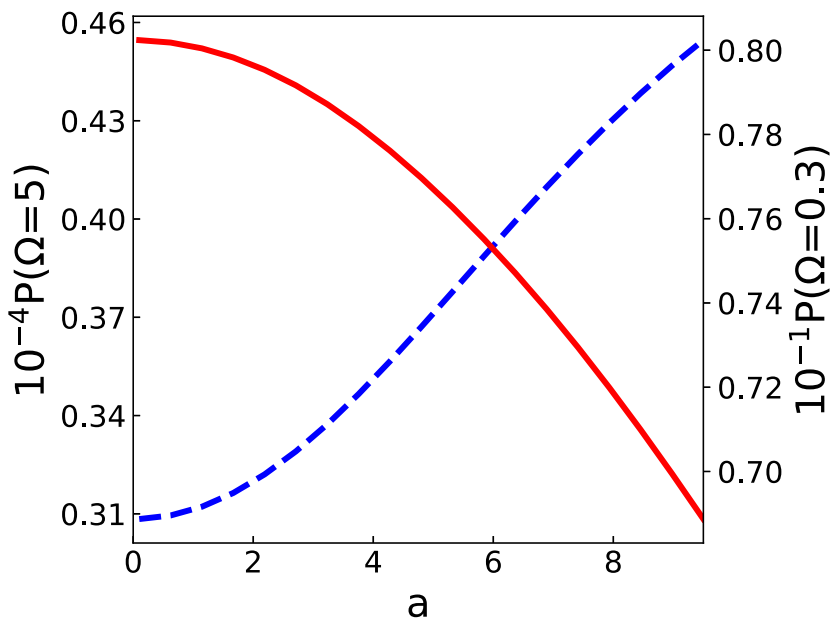

FIG. 3. The transition probability as a function of the acceleration $a$ when the atom is accelerated in the thermal field. The solid red line denotes the case that the transition probability decreases with acceleration at $\Omega=0.3$ (referenced to the right vertical axis), whereas the dashed blue line with respect to the left vertical axis is for increasing transition probability with acceleration at $\Omega=5$. The inverse of the background temperature is taken as $\beta=1$, and the other parameters are the same as in Fig. 1. conditions, which makes the atom "feel" cooler instead of warm up expected by the Unruh effect. Although the physically essential reasons remain to be explored for the difference between the anti-Unruh and Unruh effects, some important elements, like the interaction time, the detector's energy gap, the mass of the quantum field, etc, could be selected out to distinguish them operationally. See Fig. 3 for the presentation of this effect in the thermal background. Here the massive field with e.g., $\omega=$ $\sqrt{k^{2}+m^{2}}$ is considered as in Ref. [21] so that the antiUnruh effect will not be constrained by the finite interaction time and its validity can be extended to situations where the detector is switched on adiabatically over an infinite long time. Without loss of generality, $m=1$ is used for the numerical calculations. From Fig. 3, it is seen that the existence of the thermal background does not seem to affect the trend of the change for the two cases of Unruh and antiUnruh effects, although the interaction between the atom and the thermal field is more complicated than that between the atom and the vacuum. Actually, the anti-Unruh effect will disappear with the increase of the background temperature due to the disappearance of entanglement, which can be seen below with the discussions for many-body entanglement.

\section{MANY-BODY ENTANGLEMENT}

We choose the TF states as the many-body entangled quantum states and discuss the influence of acceleration on them in this section. TF states are one kind of Dicke states [45]. For a collection of $N$ identical (pseudo-) spin- $1 / 2$ particles, Dicke states can be expressed in Fock space as $\left|\frac{N}{2}+m\right\rangle_{\uparrow}\left|\frac{N}{2}-m\right\rangle_{\downarrow}$ with $\left(\frac{N}{2}+m\right)$ particles in spin-up and $\left(\frac{N}{2}-m\right)$ particles in spin-down modes for $m=-\frac{N}{2}$, $-\frac{N}{2}+1, \ldots, \frac{N}{2}$. In particular, $m=0$ represents just the TF state where the number of the particles is the same for each one of the two spin states. On the other hand, Dicke states can be described by the common eigenstate $|j, m\rangle$ of the collective spin operators $J^{2}$ and $J_{z}$, with respective eigenvalues $j(j+1)$ and $m$. For the system consisted of $N$ two-level atoms we will consider, the state $\left|j=\frac{N}{2}, m\right\rangle$ indicates that $(j+m)$ atoms are at the excited state $|e\rangle,(j-m)$ atoms are at the ground state $|g\rangle . J_{z}=$ $\frac{1}{2}\left(n_{e}-n_{g}\right)$ represents the difference of the number of atoms between excited $\left(n_{e}\right)$ and ground $\left(n_{g}\right)$ states, and $J^{2}=$ $\frac{N}{2}\left(\frac{N}{2}+1\right)$ is related to the total number of atoms. For the TF state, $m=0$ and $\left\langle J_{z}\right\rangle=0$.

The acceleration of TF states were investigated based on such consideration that the single accelerated atom was calculated according to the UDW model and all atoms were accelerated with the same way without any other interaction among atoms except their initial entanglement. Meanwhile, the distance between the atoms is much less than the relevant wavelengths of the field, which assures that all 
atoms see the same field. When all atoms in the TF state are accelerated, the state of every atom is changed. Because the excitation probability is not equal to the deexcitation probability due to the acceleration, the resulted atom's number at the excited state is not equal to that at the ground state. Thus, the final state would deviate from the TF state. In order to quantify the entanglement of the state, we choose the spin squeezing parameter

$$
\xi_{E}^{2}=\frac{(N-1)\left(\Delta J_{z}\right)^{2}+\left\langle J_{z}^{2}\right\rangle}{\left\langle J^{2}\right\rangle-N / 2} .
$$

It derives from the relation between spin squeezing and entanglement as shown in [46,47], in which the inequality $(N-1)\left(\Delta J_{\vec{n}}\right)^{2}+\left\langle J_{\vec{n}}^{2}\right\rangle \geq\left\langle J^{2}\right\rangle-N / 2$ holds for any separable states, and the violation of this inequality indicates entanglement. Here the mean-spin direction $\vec{n}$ was taken along the $z$ direction. If $\xi_{E}^{2}<1$, the state is spin squeezed and entangled. In particular, the smaller the value of $\xi_{E}^{2}$, the more the entanglement will be.

At first, we show the change of state for two accelerated atoms. It is noticed that the bipartite quantum state for the maximal entangled atoms, $\left|\psi_{i}\right\rangle=\frac{1}{\sqrt{2}}\left(|g\rangle_{A}|e\rangle_{B}+|e\rangle_{A}|g\rangle_{B}\right)$, can be regarded as the simplest TF state, i.e., $\left|\psi_{i}\right\rangle=|1,0\rangle$ using the description in the above section. Here the subscripts $A$ and $B$ in the state $\left|\psi_{i}\right\rangle$ represents the locations related to the atoms $A$ and $B$. The initial density operator is assumed to take the form

$$
\rho_{i}=\left|\psi_{i}\right\rangle\left\langle\psi_{i}\right| \otimes \rho_{t h} .
$$

For the case we consider, each atom is independently accelerating in the thermal field and has the same coupling with the field in its respective (spatial) place by the same process presented in Eq. (7). When the two atoms are accelerated simultaneously in the thermal field, the state becomes

$$
\begin{aligned}
\rho_{f}= & \operatorname{Tr}_{t h}\left[U_{A}^{\dagger} U_{B}^{\dagger} \rho_{i} U_{B} U_{A}\right] \\
= & \sum_{n_{A}, n_{B}} \frac{p_{n_{A}} p_{n_{B}}}{D}\left[\frac{1}{2}\left|u_{+}\right|^{2}\left(n_{A}+n_{B}\right)|e e\rangle\langle e e|\right. \\
& +\frac{1}{2}\left(1+n_{A}\left(n_{B}+1\right)\left|u_{+}\right|^{2}\left|u_{-}\right|^{2}\right)(|g e\rangle+|e g\rangle)(\langle g e|+\langle e g|) \\
& \left.+\frac{1}{2}\left|u_{-}\right|^{2}\left(n_{A}+n_{B}+2\right)|g g\rangle\langle g g|\right]
\end{aligned}
$$

where $|g e\rangle \equiv|g\rangle_{A}|e\rangle_{B}$ for simplicity, and $D=1+\frac{1}{2}\left|u_{+}\right|^{2}$ $\left(n_{A}+n_{B}\right)+\frac{1}{2}\left|u_{-}\right|^{2}\left(n_{A}+n_{B}+2\right)+n_{A}\left(n_{B}+1\right)\left|u_{+}\right|^{2}\left|u_{-}\right|^{2}$ is the normalization factor. Furthermore, we write the final state according to the representation for Dicke states,

$$
\begin{aligned}
\rho_{f}= & \sum_{n_{A}, n_{B}} \frac{p_{n_{A}} p_{n_{B}}}{D}\left[\frac{1}{2}\left|u_{+}\right|^{2}\left(n_{A}+n_{B}\right)|1,1\rangle\langle 1,1|\right. \\
& +\left(1+n_{A}\left(n_{B}+1\right)\left|u_{+}\right|^{2}\left|u_{-}\right|^{2}\right)|1,0\rangle\langle 1,0| \\
& \left.+\frac{1}{2}\left|u_{-}\right|^{2}\left(n_{A}+n_{B}+2\right)|1,-1\rangle\langle 1,-1|\right] .
\end{aligned}
$$

Then, the difference of the number of atoms between excited and ground states is obtained as

$$
\begin{aligned}
\left\langle J_{z}\right\rangle= & \operatorname{Tr}\left(\rho_{f} J_{z}\right) \\
= & \sum_{n_{A}, n_{B}} \frac{p_{n_{A}} p_{n_{B}}}{2 D}\left[\left|u_{+}\right|^{2}\left(n_{A}+n_{B}\right)\right. \\
& \left.-\left|u_{-}\right|^{2}\left(n_{A}+n_{B}+2\right)\right],
\end{aligned}
$$

where $\operatorname{Tr}$ represents the trace of a matrix. The result means that the atom's number at the excited state is not equal to that at the ground state, different from the requirement of TF state, unless the probability of transition from the ground state to the excited state equates the probability for the inverse transition.

We extend the discussion for two atoms to the case of $N$ atoms with the initial TF state $|j, 0\rangle$. When all atoms are accelerated simultaneously under thermal background, the TF state becomes

$$
\rho_{\mathrm{TF}}=\sum_{m=-N / 2}^{N / 2} A_{m}^{2}|j, m\rangle\langle j, m|,
$$

up to the normalization factor which is included in our numerical calculation. $A_{m}^{2}=\left[\sum_{k=0}^{N / 2-|m|} C_{N / 2}^{k} C_{N / 2}^{k+|m|}\left(P_{+} P_{-}\right)^{k}\right.$ $\left.\left(\theta(m)\left(P_{+}\right)^{m}+\theta(-m)\left(P_{-}\right)^{|m|}\right)\right]$ in which the function $\theta(x)=1$ when $x>0$ and $\theta(x)=0$ otherwise, and $C_{n}^{r}=$ $\frac{n !}{r !(n-r) !}$ denotes the combinatorial factor of choosing $r$ out of $n$. The parameter $A_{0}^{2}$ represents the probability of remaining the original form of the TF state, which includes those cases that if $l\left(0 \leq l \leq \frac{N}{2}\right)$ atoms are changed from the ground states to the excited states, there must be other $l$ atoms which are changed from the excited states to the ground states simultaneously. The reason that the second term appears is due to the inequality of the transition probabilities $P_{+}$and $P_{-}$. The parameter $A_{m}^{2}$ can be worked out by choosing the terms that in every term either there are $m$ more excited states than ground states (that is the case for $m>0$ ) or there are $m$ more ground states than excited states (that is the case for $m<0$ ). The crossed terms like $|j, m\rangle\left\langle j, m^{\prime}\right|$ have been reduced when tracing out the field degrees of freedom.

After acceleration, the TF state becomes $\rho_{\mathrm{TF}}$ described in Eq. (14). With this, we can calculate $\left\langle J_{z}\right\rangle=\operatorname{Tr}\left(\rho_{\mathrm{TF}} J_{z}\right)=$ $\sum_{m=-N / 2}^{N / 2} m A_{m}^{2}$, and $\left\langle J_{z}^{2}\right\rangle=\operatorname{Tr}\left(\rho_{\mathrm{TF}} J_{z}^{2}\right)=\sum_{m=-N / 2}^{N / 2} m^{2} A_{m}^{2}$. Thus, according to $\left(\Delta J_{z}\right)^{2}=\left\langle J_{z}^{2}\right\rangle-\left\langle J_{z}\right\rangle^{2}$, ones can 


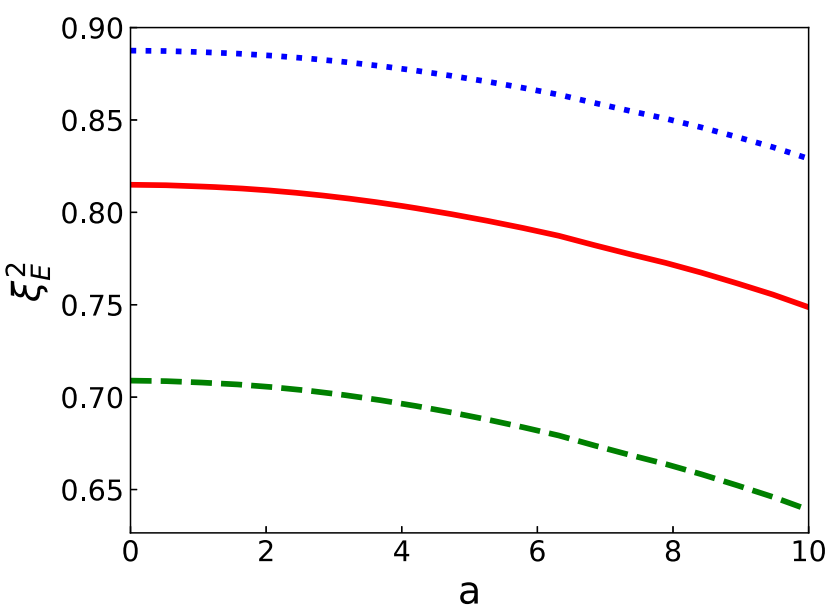

FIG. 4. The spin-squeezing parameter as a function of the acceleration $a$ when the atoms are accelerated in the thermal field. The different lines refer to different background temperatures: the dashed green line for $T=3$, the solid red line for $T=4$, and the dotted blue line for $T=5$. We make the total atom number $N=100$, and the other parameters are the same as in Fig. 1.

calculate $\xi_{E}^{2}$ by substituting these results into Eq. (9), which is presented in Fig. 4 for different background temperatures. It is seen that the spin squeezing parameters for three different background temperatures are decreasing or entanglement is increasing when the acceleration is increased, which are the indication for the anti-Unruh effect. It is noted that the entanglement at $a=0$ for the accelerated state (14) is less than that for the initial maximal entangled state due to the presence of switching function.

Moreover, we calculate the change of $\xi_{E}^{2}$ with regard to the background temperatures, which is presented in Fig. 5. It shows that the entanglement is decreasing when the background temperature increases for a fixed acceleration.

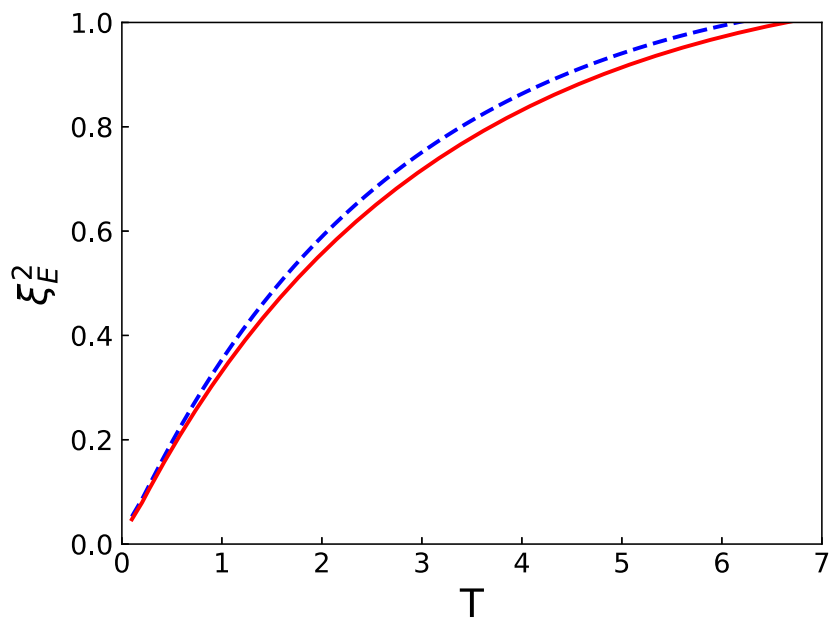

FIG. 5. The spin-squeezing parameter as a function of the background temperature when the atoms are accelerated in the thermal field. The dashed blue line is drawn at $a=2.5$, and the solid red line at $a=7.5$ We make the total atom number $N=100$, and the other parameters are the same as in Fig. 1.
When the background temperature increases to some specific value, entanglement would decrease to zero, which is similar to the sudden death of entanglement due to the increase of the temperature [29]. So, the observation of the anti-Unruh effect requires a lower background temperature, since a high background temperature would change the influence of acceleration on quantum many-body systems. Even a higher temperature would eliminate the evidence for the influence of acceleration as entanglement becomes zero.

\section{PHASE SENSITIVITY}

In this section, we continue to discuss the influence of background temperature on entanglement with the actual experimental conditions. For this purpose, we have to study the influence of acceleration on the phase sensitivity and compare it with the present experiment, since the change of spin squeezing or entanglement influences the phase sensitivity of the measurement.

Consider the Ramsey interferometer $[48,49]$ with the initial input state $\rho_{i}$, and the output state $\rho_{o}=U^{\dagger} \rho_{i} U$ where $U=\exp \left(-i \theta J_{y}\right)$ is the unitary operator for the evolution and $\theta$ is the phase shift. The phase sensitivity $\Delta \theta$ can be calculated as

$$
(\Delta \theta)_{P}^{2}=\frac{2\left(\Delta J_{z}^{2}\right)_{i}\left(\Delta J_{x}^{2}\right)_{i}+V_{x z}}{4\left(\left\langle J_{x}^{2}\right\rangle_{i}-\left\langle J_{z}^{2}\right\rangle_{i}\right)^{2}}
$$

where the subscript $i$ denotes that the average is taken under the input state and $V_{x z}=\left\langle\left(J_{x} J_{z}+J_{z} J_{x}\right)^{2}\right\rangle_{i}+\left\langle J_{z}^{2} J_{x}^{2}+\right.$ $\left.J_{x}^{2} J_{z}^{2}\right\rangle_{i}-2\left\langle J_{z}^{2}\right\rangle_{i}\left\langle J_{x}^{2}\right\rangle_{i}$. This is obtained from the error propagation formula, $(\Delta \theta)^{2}=\frac{\left(\Delta J_{z}^{2}\right)_{\theta}^{2}}{\left|d\left\langle J_{z}^{2}\right\rangle_{o} / d \theta\right|^{2}}$, by using the relation $U^{\dagger} J_{z} U=J_{z} \cos \theta-J_{x} \sin \theta$ to link the input state with the output state and by choosing the optimal phase shift through $\tan ^{2} \theta_{p}=\frac{\left(\Delta J_{z}^{2}\right)_{i}}{\left(\Delta J_{x}^{2}\right)_{i}}$. For the accelerated state in Eq. (14), a direct but tedious calculation within the approximation, $m \ll j$ and $A_{m}^{2} \ll A_{0}^{2}$ gives

$$
(\Delta \theta)_{P A}^{2} \simeq \frac{1}{2 j(j+1)}+\frac{2\left\langle J_{z}^{2}\right\rangle}{j(j+1)}+\frac{\sum_{m=-N / 2}^{N / 2} \sqrt{2} \Delta J_{z}^{2}}{2 j(j+1)},
$$

which is obtained from Eq. (15) with $\left\langle J_{z}^{2}\right\rangle=$ $\sum_{m=-N / 2}^{N / 2} m^{2} A_{m}^{2}, \quad \Delta J_{z}^{2}=\sum_{m=-N / 2}^{N / 2} m^{4} A_{m}^{2}-\left(\sum_{m=-N / 2}^{N / 2}\right.$ $\left.m^{2} A_{m}^{2}\right)^{2}, \quad\left\langle J_{x}^{2}\right\rangle=\frac{1}{2}\left[j(j+1)-\sum_{m=-N / 2}^{N / 2} m^{2} A_{m}^{2}\right], \quad \Delta J_{x}^{2} \simeq$ $\frac{1}{8} \sum_{m=-N / 2}^{N / 2} A_{m}^{2} j(j+1)\left[j(j+1)-2-8 m^{2}\right], V_{x z} \simeq \sum_{m=-N / 2}^{N / 2}$ $A_{m}^{2} m\left[(2 m+1)^{2} \alpha_{m}^{2}+(2 m-1)^{2} \beta_{m}^{2}\right]$. The calculation for $V_{x z}$ keeps to the second order of $m$ since $m$ is small, and $\alpha_{m}^{2}=\frac{1}{4}[(j-m)(j+m+1)], \beta_{m}^{2}=\frac{1}{4}[(j+m)(j-m+1)]$.

With the formula (16), we numerically calculate the condition for appearance of the anti-Unruh effect. Only if the background temperature is less than $10^{-9} \mathrm{~K}$, the antiUnruh effect can be found. This is advantageous since the 


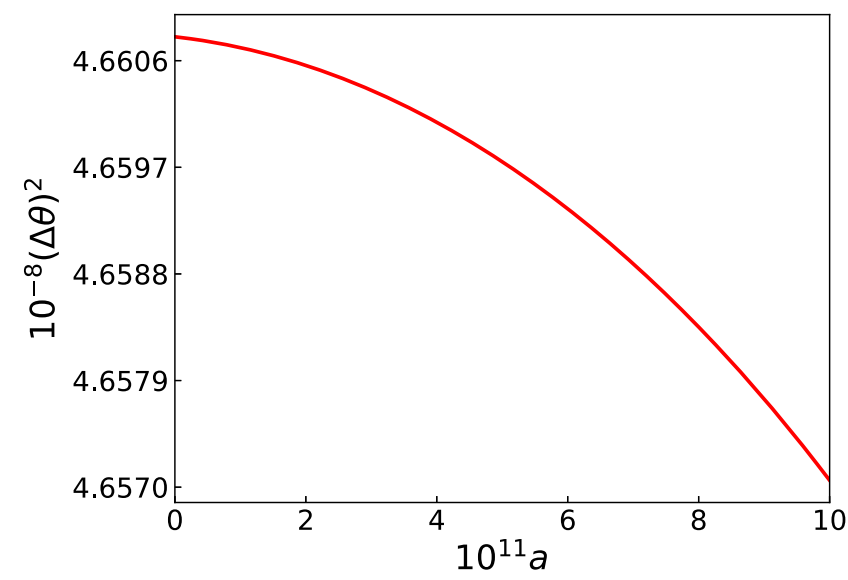

FIG. 6. The phase sensitivity as a function of the acceleration $a$. We take the parameters according to the experiment made in Ref. [44] with $\lambda=1, \sigma=26(\mu s), \Omega=2 \pi \hbar$, and $N=10000$.

temperature of $10^{-9} \mathrm{~K}$ is just required to form BoseEinstein condensates in the recent experiment that generates the TF state [44]. At the same time, it is also noted that when the acceleration reaches $10^{11} \mathrm{~m} / \mathrm{s}^{2}$, the phase sensitivity is at the level of $(\Delta \theta)_{P A}^{2} \sim 10^{-8}$, which requires a more precise experimental conditions than our earlier estimation [32] by the acceleration in the vacuum, due to the influence of the thermal field. Although it is not easy to obtain so large acceleration for Bose-Einstein condensates, the phenomena led by the background temperature is significant for any other related observations, since the antiUnruh effect gives the different behaviors from that given by the thermal background effect, in which the latter cannot lead to the increase of entanglement. This is attractive for the future experiment with higher sensitivity. Figure 6 presents the possible condition of background temperature to realize the case of anti-Unruh effect with the accelerating TF state. When the background temperature is higher than $10^{-9} \mathrm{~K}$, the anti-Unruh effect would disappear and the Bose-Einstein condensates would be broken. Similarly, the acceleration can not be larger than $10^{11} \mathrm{~m} / \mathrm{s}^{2}$ which corresponds to the Unruh temperature of $10^{-9} \mathrm{~K}$. Of course, it is expected that the observable window for the Unruh or anti-Unruh effect can occur at smaller acceleration for the future experimental technologies.

\section{CONCLUSION}

In this paper, we investigate the influence of the background temperature on the existence of anti-Unruh effect. At first, we show that for a massless scalar field the antiUnruh effect can appear for accelerated detectors in the thermal background, which is proved at the earlier time that the anti-Unruh effect is absent for accelerated detectors in the Minkowski vacuum of a massless scalar field. It is noted that the breakdown of time transitional invariance of thermal Green function is not crucial for the appearance of anti-Unruh effect. We have also calculated the change of entanglement for many-body quantum states accelerating in thermal field. It is found that the anti-Unruh effect still exists, but it will change when the background temperature increases while all other parameters are not changed. In particular, when the background temperature increases to a specific value, entanglement would decrease to zero, which wipes off any possibility to observe the influence of acceleration on entanglement irrespective of the case for the Unruh effect or the anti-Unruh effect. Finally, we have used the experimental parameters to estimate the possibility for the existence of the anti-Unruh effect. It is interesting to find that the case for anti-Unruh effect can appear for such accelerated states only if the background temperature is less than $10^{-9} \mathrm{~K}$, which is favorable for the possible future experiment since this effect is distinctive and different from that coupled to a thermal environment directly by inertial detectors.

\section{ACKNOWLEDGMENTS}

This work is supported from Grant No. 11654001 of the National Natural Science Foundation of China (NSFC).
[1] W. G. Unruh, Phys. Rev. D 14, 870 (1976).

[2] L. C. B. Crispino, A. Higuchi, and G. E. A. Matsas, Rev. Mod. Phys. 80, 787 (2008).

[3] B. S. DeWitt, S. Hawking, and W. Israel, General Relativity: An Einstein Centenary Survey (Cambridge University Press Cambridge, England, 1979).

[4] J. S. Bell and J. M. Leinaas, Nucl. Phys. B212, 131 (1983).

[5] W. G. Unruh and R. M. Wald, Phys. Rev. D 29, 1047 (1984).

[6] D. A. T. Vanzella and G. E. A. Matsas, Phys. Rev. Lett. 87, 151301 (2001).
[7] H. Suzuki and K. Yamada, Phys. Rev. D 67, 065002 (2003).

[8] R. Schutzhold, G. Schaller, and D. Habs, Phys. Rev. Lett. 97, 121302 (2006).

[9] N. Oshita, K. Yamamoto, and S. Zhang, Phys. Rev. D 93, 085016 (2016).

[10] S. Cruz, y. Cruz, and B. Mielnik, J. Phys. 698, 012002 (2016).

[11] G. Cozzella, A. G. S. Landulfo, G. E. A. Matsas, and D. A. T. Vanzella, Phys. Rev. Lett. 118, 161102 (2017).

[12] J. Rodriguez-Laguna, L. Tarruell, M. Lewenstein, and A. Celi, Phys. Rev. A 95, 013627 (2017). 
[13] M. H. Lynch, E. Cohen, Y. Hadad, and I. Kaminer, Phys. Rev. D 104, 025015 (2021).

[14] J. Hu, L. Feng, Z. Zhang, and C. Chin, Nat. Phys. 15, 785 (2019).

[15] C. A. U. Lima, F. Brito, J. A. Hoyos, and D. A. T. Vanzella, Nat. Commun. 10, 3030 (2019).

[16] M. Blasone, G. Lambiase, G. G. Luciano, and L. Petruzzielloa, Phys. Lett. B 800, 135083 (2020).

[17] W. Brenna, R. B. Mann, and E. Martín-Martínez, Phys. Lett. B 757, 307 (2016).

[18] R. Kubo, J. Phys. Soc. Jpn. 12, 570 (1957).

[19] P. C. Martin and J. Schwinger, Phys. Rev. 115, 1342 (1959).

[20] C. J. Fewster, B. A. Juárez-Aubry, and J. Louko, Classical Quantum Gravity 33, 165003 (2016).

[21] L. J. Garay, E. Martín-Martínez, and J. deRamón, Phys. Rev. D 94, 104048 (2016).

[22] I. Fuentes-Schuller and R. B. Mann, Phys. Rev. Lett. 95, 120404 (2005).

[23] P. M. Alsing, I. Fuentes-Schuller, R. B. Mann, and T. E. Tessier, Phys. Rev. A 74, 032326 (2006).

[24] E. Martín-Martínez and J. León, Phys. Rev. A 80, 042318 (2009).

[25] E. Martín-Martínez, L. J. Garay, and J. León, Phys. Rev. D 82, 064006 (2010).

[26] J. Wang and J. Jing, Phys. Rev. A 83, 022314 (2011).

[27] M. Shamirzai, B. N. Esfahani, and M. Soltani, Int. J. Theor. Phys. 51, 787 (2012).

[28] D. E. Bruschi, A. Dragan, I. Fuentes, and J. Louko, Phys. Rev. D 86, 025026 (2012).

[29] B. Richter and Y. Omar, Phys. Rev. A 92, 022334 (2015).

[30] Y. Dai, Z. Shen, and Y. Shi, J. High Energy Phys. 09 (2015) 071.

[31] T. Li, B. Zhang, and L. You, Phys. Rev. D 97, 045005 (2018).
[32] Y. Pan and B. Zhang, Phys. Rev. A 101, 062111 (2020).

[33] S. S. Costa and G. E. A. Matsas, Phys. Rev. D 52, 3466 (1995).

[34] S. Kolekar, Phys. Rev. D 89, 044036 (2014).

[35] A. P. C. M. Lima, G. Alencar, and R. R. Landim, Phys. Rev. D 101, 125008 (2020).

[36] S. Barman and B. R. Majhi, J. High Energy Phys. 03 (2021) 245.

[37] D. Barman, S. Barman, and B. R. Majhi, J. High Energy Phys. 07 (2021) 124.

[38] S. Kolekar and T. Padmanabhan, Classical Quantum Gravity 32, 202001 (2015).

[39] C. Chowdhury, S. Das, S. Dalui, and B. R. Majhi, Phys. Rev. D 99, 045021 (2019).

[40] A. P. C. M. Lima, G. Alencar, and R. R. Landim, Phys. Rev. D 101, 125008 (2020).

[41] M. Kitagawa and M. Ueda, Phys. Rev. A 47, 5138 (1993).

[42] J. Ma, X. Wang, C. Sun, and F. Nori, Phys. Rep. 509, 89 (2011).

[43] M. J. Holland and K. Burnett, Phys. Rev. Lett. 71, 1355 (1993).

[44] X. Y. Luo, Y. Q. Zou, L. N. Wu et al. Science 355, 620 (2017).

[45] R. H. Dicke, Phys. Rev. 93, 99 (1954).

[46] A. Sørensen, L. Duan, J. Cirac, and P. Zoller, Nature (London) 409, 63 (2001).

[47] G. Tóth, C. Knapp, O. Gühne, and H. J. Briegel, Phys. Rev. A 79, 042334 (2009).

[48] N. Ramsey, Molecular Beams (Oxford University Press, England, 1985).

[49] B. Yurke, S. L. McCall, and J. R. Klauder, Phys. Rev. A 33, 4033 (1986). 